\title{
Non-Locality and Late-Time Cosmic Acceleration from an Ultraviolet Complete Theory ${ }^{\dagger}$
}

\author{
Gaurav Narain ${ }^{1, *}$ and Tianjun $\mathrm{Li}^{2,3}$ \\ 1 Department of Space Science, Beihang University, Beijing 100191, China \\ 2 CAS Key Laboratory of Theoretical Physics, Institute of Theoretical Physics, Chinese Academy of Sciences, \\ Beijing 100190, China; tli@itp.ac.cn \\ 3 School of Physical Sciences, University of Chinese Academy of Sciences, No. 19A Yuquan Road, \\ Beijing 100049, China \\ * Correspondence: gaunarain@gmail.com; Tel.: +86-132-4167-0791 \\ + This paper is based on the talk at the International Conference on Quantum Gravity, Shenzhen, China, 26-28 \\ March 2018.
}

Received: 26 June 2018; Accepted: 24 July 2018; Published: 26 July 2018

\begin{abstract}
A local phenomenological model that reduces to a non-local gravitational theory giving dark energy is proposed. The non-local gravity action is known to fit the data as well as $\Lambda$-CDM thereby demanding a more fundamental local treatment. It is seen that the scale-invariant higher-derivative scalar-tensor theory of gravity, which is known to be ultraviolet perturbative renormalizable to all loops and where ghosts become innocuous, generates non-locality at low energies. The local action comprises of two real scalar fields coupled non-minimally with the higher-derivative gravity action. When one of the scalar acquiring the Vacuum Expectation Value (VEV) induces Einstein-Hilbert gravity, generates mass for fields, and gets decoupled from system, it leaves behind a residual theory which in turn leads to a non-local gravity generating dark energy effects.
\end{abstract}

Keywords: perturbative quantum field theory; cosmology; cosmological constant and dark energy; non-locality

\section{Introduction}

Dark Energy (DE) is a puzzling problem observed in the Universe at cosmological scales, where the Universe undergoes accelerated expansion [1,2]. Numerous efforts to understand the fundamental nature of it has been considered: quintessence [3-6], $\Lambda$-CDM, K-essence [7-9], but so far $\Lambda$-CDM is a winning candidate fitting the data.

It is reasonably well understood that a slowly rolling scalar gives rise to negative pressure generating dark energy effects, which is the case in quintessence model [3-6] and k-essence model [7-9]. Besides the existence of other possibilities, an interesting proposal involving non-locality has been proposed recently [10-12], where it was noticed that a particular kind of non-locality generates late-time accelerated expansion in the Universe fitting the dark-energy data as well as $\Lambda$-CDM [13,14]. However, a fundamental local explanation for it is currently lacking, although such non-localities can possibly arise as a consequence of quantum effects leading to energy dependence in coupling parameters $(g(\mu) \rightarrow g(-\square)$, where $\square$ is the square of covariant derivative while $g$ is some coupling of the theory), where the infrared behaviour of the running couplings leads to non-local modification of gravity [15,16], it was only recently [17] a model has been proposed where non-locality arises via decoupling of fields starting from a fundamental theory.

The scale-invariant higher-derivative induced gravity model coupled with scalars undergoes symmetry breaking via quantum corrections [18], thereby inducing mass for the scalar leading to its 
decoupling $[19,20]$. In a model comprising of more than one scalar, if one of the scalar gets decoupled, a phase of accelerated expansion is achieved at late times arising due to the generation of non-locality following the integration of residual scalars [11,12,14].

The outline of the paper is as follows. In Sections 2 and 3, a brief review of the non-local gravity and induced gravity, respectively, is given. Section 4 covers the non-local model of gravity, while Section 5 presents conclusions.

\section{Non-Local Gravity}

In non-local gravity, the purpose is to implement a modification in the behaviour of gravity at very large distances or late times of the Universe. The first proposal came in [21] where the General Relativity (GR) equations were phenomenologically modified to

$$
\left(1-\mu^{2} \square^{-1}\right) G_{\mu v}=8 \pi G T_{\mu v}
$$

where $\mu^{2}$ is the scale at which non-locality enters, $G_{\mu \nu}$ is the Einstein tensor for the metric, $G$ is the gravitational coupling constant, and $T_{\mu \nu}$ is the energy-momentum tensor. Lack of commutativity of $\nabla_{\mu}$ with $\square^{-1}$ on curved space-time implies non-conservation of energy-momentum tensor, indicating a much needed modification of above [22]. However, this leads to unstable cosmic evolution [23,24]. A successful non-local model, free of instabilities was given in [23]

$$
G_{\mu v}-\frac{\mu^{2}}{3}\left(g_{\mu v} \square^{-1} R\right)^{T}=8 \pi G T_{\mu \nu} .
$$

This model being devoid of Veltman-Zakharov discontinuity makes a smooth transition to GR when $\mu^{2} \rightarrow 0$. Besides having a stable cosmic evolution at various eras, the non-locality at late-times is seen to give rise to dark energy [23,24]. Furthermore, it produces a good behaviour of cosmological perturbations in scalar [25] and tensor sector [14], agrees very well with the Cosmic Microwave Background radiation, supernova, Baryon Acoustic Oscillations (BAO) and structure formation data $[25,26]$. This model named $R T$-model (where $R$ stands for Ricci Scalar while $T$ refers to transverse part) is seen to be statistically indistinguishable from $\Lambda$-CDM [13,14]. This model interestingly is closely related to the following non-local action:

$$
S_{\mathrm{NL}}=\frac{M_{P}^{2}}{2} \int \mathrm{d}^{4} x \sqrt{-g}\left[R-\frac{\mu^{2}}{6} R \square^{-2} R\right],
$$

where $M_{P}$ is the reduced Planck mass, which generates equation-of-motions (EOM) whose linearisation around flat space matches the ones obtained from Equation (2), lacking a match at the nonlinear level. This model named $R R$-model works well at background level [10], matching with data [14,25] (although it does not fit as well as $\Lambda$-CDM). Exploiting the arbitrariness associated in describing the non-local model at the level of action, it is seen that the following nonlinear extension

$$
S_{\mathrm{NL}}=\frac{M_{P}^{2}}{2} \int \mathrm{d}^{4} x \sqrt{-g}\left[R-\frac{\mu^{2}}{6} R(-\square+\xi R)^{-2} R\right],
$$

where $\xi$ is a dimensionless parameter, does a very good job in matching the data, and they get inspired from the realisation that the $R T$-model is a nonlinear extension of $R R$-model [11]. This model has been studied extensively and can fit the DE data nicely (statistically as well as $\Lambda$-CDM).

\section{Induced Gravity Model}

The induced gravity model comprises a scalar field non-minimally coupled with higher-derivative gravity, whose action is of the fourth order. The corresponding quantum theory is known to be renormalizable to all loops [27], and was recently shown to be unitary [28,29] (see also references 
therein). It then offers a sufficiently simple quantum field theory of gravity which can be used to investigate physics at ultra-high energies.

In this scale-invariant model, the scalar acquires a VEV giving rise to gravitational coupling and masses for the fields. Lack of any dimensionful parameter in the scale-invariant theory makes it perturbatively renormalizable to all loops [30]. Some of the first studies of such systems were done in [30-33], where the behaviour of parameters under radiative corrections were done.

Recently, this topic has gained renewed interest [34-38]. Here, a scale is generated dynamically, starting from a scale-invariant system by transforming the system from Jordon to Einstein frame where the potential acquires a VEV resulting in scale-generation. However, unitarity is not explored ${ }^{1}$ It is seen in [18] that the Jordan frame theory under quantum corrections induce ghost mass that is always above energy scale, thereby making it innocuous. The renormalizable and UV well defined scale-invariant action that one considers here is

$$
S_{\mathrm{GR}}=\int \mathrm{d}^{4} x \sqrt{-g}\left[\frac{1}{16 \pi}\left\{-\frac{1}{f^{2}}\left(R_{\mu \nu} R^{\mu \nu}-\frac{1}{3} R^{2}\right)+\frac{\omega R^{2}}{6 f^{2}}\right\}+\frac{1}{2} \partial_{\mu} \phi \partial^{\mu} \phi-\frac{\lambda}{4} \phi^{4}-\frac{\xi}{2} R \phi^{2}\right],
$$

where the coupling $f^{2}, \omega, \lambda$ and $\xi$ are all dimensionless, while the curvature and covariant-derivative depend on metric $g_{\mu v}$. Decomposing the metric $g_{\mu \nu}=\bar{g}_{\mu v}+h_{\mu \nu}$ (where $\bar{g}_{\mu v}$ is background and $h_{\mu \nu}$ is fluctuation) leads to propagation of the fluctuation field $h_{\mu v}$ and its various couplings. On flat space-time, the propagator of quantum metric fluctuations in momentum space (for the Landau gauge condition $\partial^{\mu} h_{\mu v}=0$ ) is given by,

$$
D^{\mu v \rho \sigma}=\left(\Delta_{G}^{-1}\right)^{\mu v \rho \sigma}=(16 \pi) \frac{f^{2}}{q^{4}}\left(-2 P_{2}^{\mu v \rho \sigma}+\frac{1}{\omega} P_{s}^{\mu v \rho \sigma}\right),
$$

where $\left(P_{2}\right)_{\mu \nu}{ }^{\alpha \beta}=\left[T_{\mu}{ }^{\alpha} T_{v}{ }^{\beta}+T_{\mu}{ }^{\beta} T_{v}{ }^{\alpha}\right] / 2-(1 /(d-1)) T_{\mu v} T^{\alpha \beta}$ and $\left(P_{s}\right)_{\mu \nu}{ }^{\alpha \beta}=(1 /(d-1)) T_{\mu \nu} T^{\alpha \beta}$ are spin projectors, while $L_{\mu \nu}=q_{\mu} q_{\nu} / q^{2}$ and $T_{\mu \nu}=\eta_{\mu \nu}-q_{\mu} q_{\nu} / q^{2}$. The coupling signs are chosen such that tachyons do not get generated after symmetry breaking [18], which implies $f^{2}>0, \omega>0, \lambda>0$ and $\xi>0$ (for the details on this choice of signs, see $[18,40]$ ). VEV generated via quantum corrections leads to generation of mass $m_{s}^{2}=3 / 2 \lambda \kappa^{2}$ and an effective Newton's constant $G^{-1}=8 \pi \xi \kappa^{2}$ with the right sign. The graviton propagator after the symmetry breaking is as follows [18]:

$$
D^{\mu v, \alpha \beta}=16 \pi G\left[\frac{\left(2 P_{2}-P_{S}\right)^{\mu v, \alpha \beta}}{q^{2}+i \epsilon}+\frac{\left(P_{S}\right)^{\mu v, \alpha \beta}}{q^{2}-M^{2} / \omega+i \epsilon}-\frac{2\left(P_{2}\right)^{\mu v, \alpha \beta}}{q^{2}-M^{2}+i \epsilon}\right],
$$

where the masses $M^{2}=8 \pi f^{2} \xi \kappa^{2}$ and $M^{2} / \omega=8 \pi f^{2} \xi \kappa^{2} / \omega$. It clearly shows the instability caused if $\omega<0$ due to generation of tachyons $[18,28,29,40]$. The last term of the propagator in the broken phase (7) has an incorrect sign leading to trouble with unitarity, which is a consequence of higher-derivatives. If the generated mass of this ghost is such thats its mass always remains above the energy scale, then it becomes innocuous. Pure higher-derivative gravity without matter [28,29] indeed witnesses such a phenomena making the ghost innocuous for a large domain of coupling parameter space. This is repeated for the case of induced gravity [18]. However, in the case of induced gravity, it is furthermore seen that the scalar that acquires a VEV gets decoupled from the system in the same manner as for the higher-derivative ghost. Such a decoupling implies natural consequences in cosmology.

1 In [39], a quantum mechanical treatment of higher-derivative theories is attempted, whose suitable generalisation is expected to offer a treatment of ghosts in higher-derivative gravity. 


\section{Local to Non-Local}

The kind of non-local action stated in Equation (4) is seen to naturally arise in a generalised induced gravity scenario [17]. In induced gravity scenario [17], the scalar responsible for generating $\mathrm{VEV}$, mass scale, and Newton's constant gets decoupled from the system when the higher-derivative ghosts are evicted. Therefore, such decoupling can be exploited to construct models giving rise to non-localities in deep infrared. A two-scalar field model coupled non-minimally to gravity is considered

$$
S=\int \mathrm{d}^{4} x \sqrt{-g}\left[\frac{1}{16 \pi}\left\{-\frac{1}{f^{2}}\left(R_{\mu \nu} R^{\mu \nu}-\frac{1}{3} R^{2}\right)+\frac{\omega R^{2}}{6 f^{2}}\right\}+\frac{R}{2} \Phi^{T} \xi \Phi+\frac{1}{2} \Phi^{T}(-\square) \Phi-V\left(\Phi^{T} \Phi\right)\right],
$$

where $\Phi=\{\phi, \chi\}$ is a two real scalar field doublet. It is an extension of model stated in Equation (5), where the signs of parameters are taken as before to ensure stability and avoid tachyons. The dimensionless parameter $\xi$ is a matrix whose entries are given by $\xi=\left\{\left\{\xi_{1}, \xi_{12}\right\},\left\{\xi_{21}, \xi_{2}\right\}\right\}$. This is a renormalizable scale-invariant action $[27,30]$, where the addition of an extra scalar doesn't hinder the well-defined ultraviolet behaviour of theory and unitarity [18]. The $\phi^{4}$ form of potential is considered as it obeys scale symmetry and renormalizability. This means $V\left(\Phi^{T} \Phi\right)=\frac{1}{4}\left(\Phi^{T} \lambda \Phi\right)^{2}$, where the coupling matrix $\lambda=\left\{\left\{\lambda_{1}, \lambda_{12}\right\},\left\{\lambda_{21}, \lambda_{2}\right\}\right\}$ consist of only dimensionless entries. Here, one of the scalar $\phi$ acquires VEV following the Coleman-Weinberg procedure as in [18], which leads to generation of masses and effective Newton's constant. The fluctuations around VEV denoted by $\varphi$ get decoupled from the system eventually, which is noticed from renormalisation group evolution [18]. The fluctuation field $\varphi$ couples with the other scalar field $\chi$ and its action is given by

$$
\begin{aligned}
S= & \frac{1}{2} \int \mathrm{d}^{4} x \sqrt{-g}\left[\kappa^{2} \xi_{1} R+2 \kappa \xi_{1} \varphi R+\varphi\left(-\square+\xi_{1} R\right) \varphi+\kappa\left(\xi_{12}+\xi_{21}\right) R \chi+\varphi\left(-2 \square+\xi_{12} R+\xi_{21} R\right) \chi\right. \\
& \left.+\chi\left(-\square+\xi_{2} R\right) \chi-\frac{1}{2}\left\{\lambda_{1} \kappa^{2}+2 \lambda_{1} \kappa \varphi+\left(\lambda_{12}+\lambda_{21}\right) \kappa \chi+\lambda_{1} \varphi^{2}+\left(\lambda_{12}+\lambda_{21}\right) \varphi \chi+\lambda_{2} \chi^{2}\right\}^{2}\right] .
\end{aligned}
$$

In a special scenario when $\left(\lambda_{12}+\lambda_{21}\right)=0$, many terms disappear leading to a simplified form. In this special case, mixing disappears with the induced mass for the fields $\varphi$ and $\chi$ given by $m_{1}^{2}=3 \lambda_{1}^{2} \kappa^{2}$ and $m_{2}^{2}=\lambda_{1} \lambda_{2} \kappa^{2}$, respectively. The interaction piece in this special case is

$$
I=-\frac{1}{4} \lambda_{1}^{2} \kappa^{4}-\lambda_{1}^{2} \kappa^{3} \varphi-\lambda_{1} \kappa \varphi\left\{\lambda_{1} \varphi^{2}+\lambda_{2} \chi^{2}\right\}-\frac{1}{4}\left\{\lambda_{1}^{2} \varphi^{4}+2 \lambda_{1} \lambda_{2} \varphi^{2} \chi^{2}+\lambda_{2}^{2} \chi^{4}\right\}
$$

Following [17], the dynamical evolution equations for fields $\varphi$ (fluctuation) and $\chi$ is obtained by varying the full residual action. Although these are coupled nonlinear differential equations, they achieve simplification with the decoupling of scalar $\varphi$ and for small interaction strengths (which is the case in cosmological scenarios). In scale-invariant higher-derivative gravity Equation (5), this phenomena naturally occurs when scale-symmetry gets broken. In this case, the induced mass of the scalar is always above the energy scale leading to its decoupling from the system [18]. This implies $m_{1}^{2} / E^{2}>1$ ( $E$ is the running energy), which physically means that the particle does not go on-shell. In deep infrared, it is seen that this ratio $m_{1}^{2} / E^{2} \gg 1$ gets translated to $m_{1}^{2} \gg \square$. This offers simplification in our system of equations:

$$
\begin{aligned}
& \varphi=-\frac{1}{3} \kappa-\frac{1}{m_{1}^{2}}\left(\square \chi-\frac{\left(\xi_{12}+\xi_{21}\right) R \chi}{2}\right), \\
& \left(-\square+\xi_{2} R-\frac{4}{9} m_{2}^{2}\right) \chi+\frac{\left(\xi_{12}+\xi_{21}\right) R}{3} \kappa=0,
\end{aligned}
$$

where we have ignored nonlinear interactions that are higher-order. The second equation can be easily solved after inverting the operator. This then appears as a constraint in the system after decoupling of the $\varphi$ has occurred. Plugging back the solution for $\varphi$ from Equation (11) in the action generates 
leading and sub-leading terms. The sub-leading part (which is of $\left.\mathrm{O}\left(1 / m_{1}^{2}\right)\right)$ can be safely ignored under decoupling. To leading order, the action is given by

$$
S=\int \mathrm{d}^{4} x \sqrt{-g}\left[-\frac{4}{81} m_{1}^{2} \kappa^{2}+\frac{2 \xi_{1}}{9} \kappa^{2} R+\frac{\xi_{12}+\xi_{21}}{3} \kappa R \chi+\frac{1}{2}(\partial \chi)^{2}+\frac{\xi_{2}}{2} R \chi^{2}-\frac{2}{9} m_{2}^{2} \chi^{2}-\frac{1}{4} \lambda_{2}^{2} \chi^{4}\right] .
$$

Decoupling results in a residual action for field $\chi$, the matter field $\chi$ couples non-minimally with the with the background space-time. Although symmetry breaking generates a large cosmological constant, but its effects gets shielded under the vanishing of gravitational coupling in infrared, which happens in induced gravity coupled with higher-derivatives (see Equation (73), Figures 7, 11 and 13 of [18]). Even in pure higher-derivative gravity [28,29] (and in case of gravity coupled with gauge fields [41,42]), such vanishing of gravitational coupling is seen. Moreover, the field $\chi$ slow rolls when coupling $\lambda_{2}$ and mass $m_{2}^{2}$ is small, leading to ignoring of the kinetic term of the field $\chi$. The field $\chi$ no longer has dynamics in this slow-roll regime. Its coupling with the background picture drives the dynamics of space-time. In this, if the following approximation holds,

$$
\frac{2 \xi_{1}}{3\left(\xi_{12}+\xi_{21}\right)} \gg \frac{\chi}{\kappa} \gg \frac{2\left(\xi_{12}+\xi_{21}\right)}{3 \xi_{2}},
$$

then the linear $\chi$ term contribution gets suppressed by contributions from quadratic and quartic piece in $\chi$, and the induced Einstein-Hilbert piece [17]. This leaves us with a reduced action for field $\chi$ :

$$
S=\int \mathrm{d}^{4} x \sqrt{-g}\left[\frac{2 \xi_{1}}{9} \kappa^{2} R+\frac{\xi_{2}}{2} R \chi^{2}-\frac{2}{9} m_{2}^{2} \chi^{2}-\frac{1}{4} \lambda_{2}^{2} \chi^{4}\right]
$$

This action, however, comes with constraint Equation (12). Plugging back this constraint in this residual action leads to a non-local version of the theory

$$
\begin{aligned}
S_{\mathrm{NL}}= & \int \mathrm{d}^{4} x \sqrt{-g}\left[\frac{2 \xi_{1}}{9} \kappa^{2} R-\frac{2 m_{2}^{2} \kappa^{2}\left(\xi_{12}+\xi_{21}\right)^{2}}{81} R\left(-\square+\xi_{2} R-\frac{4}{9} m_{2}^{2}\right)^{-2} R+\frac{\xi_{2} \kappa^{2}\left(\xi_{12}+\xi_{21}\right)^{2} R}{18}\right. \\
& \left.\times\left\{\left(-\square+\xi_{2} R-\frac{4}{9} m_{2}^{2}\right)^{-1} R\right\}^{2}-\frac{\lambda_{2}^{2} \kappa^{4}\left(\xi_{12}+\xi_{21}\right)^{4}}{324}\left\{\left(-\square+\xi_{2} R-\frac{4}{9} m_{2}^{2}\right)^{-1} R\right\}^{4}\right] .
\end{aligned}
$$

This is a low energy gravitational action where the first term is the induced gravitational term that controls the dynamics at astronomical scales agreeing with Einstein-Hilbert gravity while other terms are generated under decoupling and approximations. These are non-local gravitational interactions dictating the behaviour of gravitational interactions at large cosmological scales. It can be understood as a heuristic derivation of the non-local action that has been studied extensively in $[11,12,14]$ and is seen to agree with the various cosmological data as good as $\Lambda$-CDM. Defining the induced Newton's constant as $9 /(32 \pi G)^{-1}=\xi_{1} \kappa^{2}$, and pulling out the factor of Planck's mass from the action allows for making comparison with the existing models in $[11,12,14]$. This will become

$$
\begin{aligned}
& S_{\mathrm{NL}}=\frac{M_{p}^{2}}{2} \int \mathrm{d}^{4} x \sqrt{-g}\left[R-\frac{\mu^{2}}{6} R\left(-\square+\xi_{2} R-\frac{4}{9} m_{2}^{2}\right)^{-2} R\right. \\
& \left.+\rho_{1}^{2} R\left\{\left(-\square+\xi_{2} R-\frac{4}{9} m_{2}^{2}\right)^{-1} R\right\}^{2}-\rho_{2}^{2}\left\{\left(-\square+\xi_{2} R-\frac{4}{9} m_{2}^{2}\right)^{-1} R\right\}^{4}\right],
\end{aligned}
$$

where $M_{P}^{2}=\left(4 \xi_{1} \kappa^{2}\right) / 9$ is the reduced Planck's mass and

$$
\mu^{2}=\frac{2\left(\xi_{12}+\xi_{21}\right)^{2} m_{2}^{2}}{3 \xi_{1}}, \quad \rho_{1}^{2}=\frac{\xi_{2}\left(\xi_{12}+\xi_{21}\right)^{2}}{4 \xi_{1}}, \quad \rho_{2}^{2}=\frac{\lambda_{2}^{2} M_{P}^{2}\left(\xi_{12}+\xi_{21}\right)^{4}}{32 \xi_{1}^{2}}
$$


are the parameters defined to make connection with the original non-local model defined in Equation (4). As seen from $[11,12,14]$, the mass scale $\mu$ should be quite small (of the $\mathcal{O}\left(H_{0}\right)$, which is $\left.\mathcal{O}\left(10^{-32}\right) \mathrm{eV}\right)$ in order to explain current accelerated expansion of the Universe. The authors of these papers further mentions that the fundamental length scale that enters the physics system is $\Lambda_{R R}$ and is related to $\mu^{2}$ via $M_{P}^{2}$ as $\Lambda_{R R}^{4}=M_{P}^{2} \mu^{2} / 12$. For our present case, this implies that $\Lambda_{R R}$ is related to the $m_{2}$ and $\kappa$ in the following way:

$$
\Lambda_{R R}^{4}=\frac{2}{81}\left(\xi_{12}+\xi_{21}\right)^{2} m_{2}^{2} \kappa^{2} .
$$

This means that if $\mu \sim \mathcal{O}\left(H_{0}\right)$, then $\Lambda_{R R} \sim 10^{-3} \mathrm{eV}$. In well known grand unified theory (GUT) models, if the scale symmetry breaks around GUT scale resulting in generation of induced Newton's constant, then this will imply that VEV $\kappa \sim 10^{16} \mathrm{GeV}$ and correspondingly $\xi_{1} \sim 100$. The mass of the scalar $\chi$ can be extracted following Equation (19), which is $m_{2} \sim 10^{-30}\left(\xi_{12}+\xi_{21}\right)^{-1} \mathrm{eV}$. Renormalizability, scale-invariance and unitarity offer sufficient freedom to choose parameters $\left(\xi_{12}+\xi_{21}\right)$ allowing one to have a reasonable $m_{2}$. This generalised induced gravity model that has well-defined UV completion and ghosts that are innocuous can then be seen to offer an interesting picture low-energy picture, where non-local interaction emerges in deep infrared leading to accelerated expansion at cosmological scales.

\section{Conclusions}

An attempt has been made in order to understand dark energy which is causing the accelerated expansion of the universe. A local model is presented which is an ultraviolet well behaved Quantum Field Theory. It is a scale-invariant, perturbatively renormalizable model which is devoid of tachyons and where ghosts have been eradicated $[18,28,29,31,43]$. A two scalar-field model is considered which couples non-minimally with the scale-invariant higher-derivative gravity. One of the scalars develops a VEV and in turn induces low energy Einstein gravity and masses for fields. This scalar eventually decouples from the system as it becomes very massive in infrared and leaves behind a simplified system of residual scalar field coupled non-minimally with gravity. This residual local field theory model leads to non-local gravity action whose leading term matches the non-local gravity action studied extensively by $[11-14,26]$ to offer an alternative explanation for dark energy. This local scale-invariant system then offers a very nice explanation for the non-local gravity considered in [11-14,26]. The local model is not only scale invariant but also, being renormalizable and unitary, has a well defined UV completion. Furthermore, it offers a "meaning" to the parameter $\mu^{2}$ (or $\Lambda_{R R}$ ) and gives a possible explanation for them.

The purpose of this work is to offer a possible local explanation for the non-local model considered by $[11-14,26]$. This non-local model has been verified against various cosmological data sets, and is seen to be as good as $\Lambda$-CDM. This motivated us to seek a possible UV complete local theory which results in this particular non-local gravity action. Having succeeded in finding such a local theory, it is now important to do a more detailed study of the same. This involves, for example, investigating duration of the radiation/matter dominated era and other cosmological scenarios, which will be done in future work.

Author Contributions: The authors contributed equally to this work.

Funding: This research received no external funding.

Acknowledgments: G.N. and T.L. would like to thank the organisers Leonardo Modesto, Casimo Bambi, and Gianluca Calcagni for organising a wonderful conference at SUSTech. G.N. is very grateful to the organisers for generously supporting his participation in the conference.

Conflicts of Interest: The authors declare no conflicts of interest. 


\section{References}

1. Riess, A.G.; Filippenko, A.V.; Challis, P.; Clocchiatti, A.; Diercks, A.; Garnavich, P.M.; Gilliland, R.L.; Hogan, C.J.; Jha, S.; Kirshner, R.P.; et al. Observational evidence from supernovae for an accelerating universe and a cosmological constant. Astron. J. 1998, 116, 1009-1083. [CrossRef]

2. Perlmutter, S.; Aldering, G.; Goldhaber, G.; Knop, R.A.; Nugent, P.; Castro, P.G.; Deustua, S.; Fabbro, S.; Goobar, A.; Groom, D.E.; et al. Measurements of Omega and Lambda from 42 high redshift supernovae. Astron. J. 1999, 517, 565-586. [CrossRef]

3. Wetterich, C. Cosmology and the Fate of Dilatation Symmetry. Nucl. Phys. B 1988, 302, 668-696. [CrossRef]

4. Frieman, J.A.; Hill, C.T.; Stebbins, A.; Waga, I. Cosmology with ultralight pseudo Nambu-Goldstone bosons. Phys. Rev. Lett. 1995, 75, 2077-2080. [CrossRef] [PubMed]

5. Zlatev, I.; Wang, L.M.; Steinhardt, P.J. Quintessence, cosmic coincidence, and the cosmological constant. Phys. Rev. Lett. 1999, 82, 896-899. [CrossRef]

6. Ferreira, P.G.; Joyce, M. Structure formation with a selftuning scalar field. Phys. Rev. Lett. 1997, 79, 4740-4743. [CrossRef]

7. Garriga, J.; Mukhanov, V.F. Perturbations in k-inflation. Phys. Lett. B 1999, 458, 219-225. [CrossRef]

8. Armendariz-Picon, C.; Damour, T.; Mukhanov, V.F. k-inflation. Phys. Lett. B 1999, 458, 209-218. [CrossRef]

9. Armendariz-Picon, C.; Mukhanov, V.F.; Steinhardt, P.J. A Dynamical solution to the problem of a small cosmological constant and late time cosmic acceleration. Phys. Rev. Lett. 2000, 85, 4438-4441. [CrossRef] [PubMed]

10. Maggiore, M.; Mancarella, M. Nonlocal gravity and dark energy. Phys. Rev. D 2014, 90, 023005. [CrossRef]

11. Cusin, G.; Foffa, S.; Maggiore, M.; Mancarella, M. Conformal symmetry and nonlinear extensions of nonlocal gravity. Phys. Rev. D 2016, 93, 083008. [CrossRef]

12. Maggiore, M. Nonlocal Infrared Modifications of Gravity. A Review. Fundam. Theor. Phys. 2017, 187, 221-281, doi:10.1007/978-3-319-51700-116. [CrossRef]

13. Dirian, Y.; Foffa, S.; Kunz, M.; Maggiore, M.; Pettorino, V. Non-local gravity and comparison with observational datasets. J. Cosmol. Astropart. Phys. 2015, 1504, 044. [CrossRef]

14. Dirian, Y.; Foffa, S.; Kunz, M.; Maggiore, M.; Pettorino, V. Non-local gravity and comparison with observational datasets. II. Updated results and Bayesian model comparison with $\Lambda$ CDM. J. Cosmol. Astropart. Phys. 2016, 1605, 068. [CrossRef]

15. Maggiore, M. Dark energy and dimensional transmutation in $R^{2}$ gravity. arXiv, 2017, arXiv:1506.06217.

16. Maggiore, M. Perturbative loop corrections and nonlocal gravity. Phys. Rev. D 2016, 93, 063008. [CrossRef]

17. Narain, G.; Li, T. Ultraviolet complete dark energy model. Phys. Rev. D 2018, 97, 083523. [CrossRef]

18. Narain, G. Exorcising Ghosts in Induced Gravity. Eur. Phys. J. C 2017, 77, 683. [CrossRef]

19. Appelquist, T.; Carazzone, J. Infrared Singularities and Massive Fields. Phys. Rev. D 1975, 11, $2856-2861$. [CrossRef]

20. Gorbar, E.V.; Shapiro, I.L. Renormalization group and decoupling in curved space. J. High Energy Phys. 2003, 0302, 021. [CrossRef]

21. Arkani-Hamed, N.; Dimopoulos, S.; Dvali, G.; Gabadadze, G. Non-local modification of gravity and the cosmological constant problem. arXiv, 2017, arXiv:hep-th/0209227.

22. Jaccard, M.; Maggiore, M.; Mitsou, E. Nonlocal theory of massive gravity Phys. Rev. D 2013, 88, 044033. [CrossRef]

23. Maggiore, M. Phantom dark energy from nonlocal infrared modifications of general relativity. Phys. Rev. D 2014, 89, 043008. [CrossRef]

24. Foffa, S.; Maggiore, M.; Mitsou, E. Cosmological dynamics and dark energy from nonlocal infrared modifications of gravity. Int. J. Mod. Phys. A 2014, 29, 1450116. [CrossRef]

25. Dirian, Y.; Foffa, S.; Khosravi, N.; Kunz, M.; Maggiore, M. Cosmological perturbations and structure formation in nonlocal infrared modifications of general relativity. J. Cosmol. Astropart. Phys. 2014, 1406, 033. [CrossRef]

26. Barreira, A.; Li, B.; Hellwing, W.A.; Baugh, C.M.; Pascoli, S. Nonlinear structure formation in Nonlocal Gravity. J. Cosmol. Astropart. Phys. 2014, 1409, 031. [CrossRef]

27. Stelle, K.S. Renormalization of Higher Derivative Quantum Gravity. Phys. Rev. D 1977, 16, 953-969. [CrossRef]

28. Narain, G.; Anishetty, R. Short Distance Freedom of Quantum Gravity. Phys. Lett. B 2012, 711, $128-131$. [CrossRef] 
29. Narain, G.; Anishetty, R. Unitary and Renormalizable Theory of Higher Derivative Gravity. J. Phys. Conf. Ser. 2012, 405, 012024. [CrossRef]

30. Fradkin, E.S.; Tseytlin, A.A. Renormalizable asymptotically free quantum theory of gravity. Nucl. Phys. B 1982, 201, 469-491. [CrossRef]

31. Julve, J.; Tonin, M. Quantum Gravity with Higher Derivative Terms. Nuovo Cim. B 1978, 46, $137-152$. [CrossRef]

32. Barth, N.H.; Christensen, S.M. Quantizing Fourth Order Gravity Theories. 1. The Functional Integral. Phys. Rev. D 1983, 28, 1876-1893. [CrossRef]

33. Avramidi, I.G.; Barvinsky, A.O. Asymptotic Freedom in Higher Derivative Quantum Gravity. Phys. Lett. B 1985, 159, 269-274. [CrossRef]

34. Salvio, A.; Strumia, A. Agravity. J. High Energy Phys. 2014, 1406, 080. [CrossRef]

35. Einhorn, M.B.; Jones, D.R.T. Naturalness and Dimensional Transmutation in Classically Scale-Invariant Gravity. J. High Energy Phys. 2015, 1503, 047. [CrossRef]

36. Jones, T.; Einhorn, M. Quantum Gravity and Dimensional Transmutation. PoS PLANCK 2015, 2015, 061,

37. Einhorn, M.B.; Jones, D.R.T. Induced Gravity I: Real Scalar Field. J. High Energy Phys. 2016, 1601, 019. [CrossRef]

38. Einhorn, M.B.; Jones, D.R.T. Induced Gravity II: Grand Unification. J. High Energy Phys. 2016, $1605,185$. [CrossRef]

39. Salvio, A.; Strumia, A. Quantum mechanics of 4-derivative theories. Eur. Phys. J. C 2016, 76, 227. [CrossRef] [PubMed]

40. Narain, G. Signs and Stability in Higher-Derivative Gravity. Int. J. Mod. Phys. A 2018, 33, 1850031. [CrossRef]

41. Narain, G.; Anishetty, R. Charge Renormalization due to Graviton Loops. J. High Energy Phys. 2013, $1307,106$. [CrossRef]

42. Narain, G.; Anishetty, R. Running Couplings in Quantum Theory of Gravity Coupled with Gauge Fields. J. High Energy Phys. 2013, 1310, 203. [CrossRef]

43. Salam, A.; Strathdee, J.A. Remarks on High-energy Stability and Renormalizability of Gravity Theory. Phys. Rev. D 1978, 18, 4480. [CrossRef]

(C) 2018 by the authors. Licensee MDPI, Basel, Switzerland. This article is an open access article distributed under the terms and conditions of the Creative Commons Attribution (CC BY) license (http://creativecommons.org/licenses/by/4.0/). 